GW23-e2322 EVALUATION OF HIGH-FREQUENCY

\title{
ULTRASONOGRAPHY ON THE IMPACT OF
} GLYCOSYLATED HAEMOGLOBIN ON CAROTID INTIMAMEDIAL THICKNESS AND HAEMODYNAMICS OF NEWLY DIAGNOSED TYPE2 DIABETES MELLITUS PATIENTS

doi:10.1136/heartjnl-2012-302920ad.47

${ }^{1}$ Wang Ping, ${ }^{1}$ Zhang Er Hong, ${ }^{2}$ Li Guang Xia, ${ }^{1}$ Wang Ping. ${ }^{1}$ The Third Affiliated Hospital of Sun Yat-Sen University; ${ }^{2} \mathrm{Da}$ Qing Long Nan Hospital

Objectives To explore the value of high-frequency ultrasonography (US) for evaluating the impact of different levels of glycosylated haemoglobin (HbA1c) on carotid intima-medial thickness (IMT) and haemodynamics of newly diagnosed type 2 diabetes mellitus (T2DM) patients.

Methods Ninety newly diagnosed T2DM patients were divided into 2 groups (Group A and Group B) according to levels of $\mathrm{HbA1c}$ (Group A, HbA1c<6.5\%; Group B, HbA1c > =6.5\%), whereas 60 healthy volunteers as controls in the Group C. High-frequency US examinations were performed to evaluate the IMT, arterial plaque and haemodynamics parameters of bilateral carotid arteries of each participant.

Results Both Group A and Group B had significantly thicker IMT than Group C (both $\mathrm{p}<0.05$ ), and the IMT of Group B was thicker

than that of Group A $(\mathrm{p}<0.05)$. The incidence of arterial plaque and plaque score were significantly higher in Group B than in Group A and Group C (both $p<0.05$ ), but there was no difference between the latter two groups $(p>0.05)$. There were significant difference of haemodynamics parameters between Group $\mathrm{A}$ and Group C and between Group B and Group C (both $p<0.05)$, while no difference between Group A and Group B ( $p>0.05)$.

Conclusions High-frequency ultrasound can be used to evaluate the IMT, arterial plaque and haemodynamics of carotid arteries of newly diagnosed T2DM patients with different levels of HbA1c, which provide early diagnosis of carotid complication due to T2DM. 